\title{
5-Alpha-Reduktase-Hemmer zur PCa-Prophylaxe nutzlos
}

— Bislang gibt es kein zugelassenes Medikament zur Prophylaxe gegen Prostatakrebs. Wenn es nach einem Beratungsgremium der US-Zulassungsbehörde FDA (Food and Drug Administration) geht, wird sich daran vorerst nichts ändern: In ihrem Urteil zu einer potenziellen Zulassungserweiterung der 5-Alpha-Reduktase-Hemmer Dutasterid (Avodart ${ }^{\circledR}$ ) und Finasterid (Proscar ${ }^{\circledR}$ ) stimmten die Mitglieder des Gremiums mit 14:2 beziehungsweise 17:0 dagegen, die beiden Substanzen zur Prävention des Prostatakarzinoms zuzulassen.

Bisher sind die beiden 5-Alpha-Reduktase-Inhibitoren zur Behandlung der Benignen Prostatahyperplasie (BPH) zugelassen. Avodart $^{\circledR}$-Hersteller GlaxoSmithKline strebt eine Zulassungserweiterung zur Behandlung von Männern mit erhöhtem PSA-Wert und negativer Biopsie an. Zwar konnte in Studien gezeigt werden, dass die 5-Alpha-Reduktase-Hemmer die Entstehung von auf die Prostata beschränkten Niedrig-Risiko-Prostatakarzinomen besser verhindern als Placebo - doch ebenso wurden beide Substanzen mit der Entstehung von metastasierten Hoch-Risiko-Prostatakarzinomen in Verbindung gebracht. Das Gremium einigte sich darauf, dass der Nutzen, vor lokal beschränkten Tumoren zu schützen, die womöglich nie metastasieren würden, nicht ausreiche, wenn die
Wirkstoffe potenziell zu Hoch-Risiko-Karzinomen führen könnten. „Wenn man gesunden Menschen ein Medikament verabreicht, darf das keinerlei Nebenwirkungen haben“, erklärte Dr. Patrick Walsh die Entscheidung des FDA-Gremiums. Ein FDA-Statistiker rechnete vor, dass von 200 Männern, die mit einem der beiden Stoffe behandelt werden würden, einer zusätzlich einen Tumor mit einem Gleason-Score 8-10 bekommen würde. Gleichzeitig müssten 60 Männer einen der beiden 5-Alpha-ReduktaseHemmer verabreicht bekommen, um einen relevanten Tumor zu verhindern.

Sowohl GlaxoSmithKline als auch Proscar ${ }^{\circledR}$-Hersteller MSD argumentierten, dass ihre Arzneimittel keine Hoch-Risiko-Prostatakarzinome verursachen und lieferten in der Sitzung andere Gründe, warum in den Studien Patienten auffällig häufig metastasierte Tumoren hatten - doch ohne Erfolg. GlaxoSmithKline bedauerte die Entscheidung des Gremiums und plant, im weiteren Verfahren eng mit der FDA zusammenzuarbeiten, um doch noch die Zulassungserweiterung zu bekommen.

$\mathrm{Ob}$ das gelingt, ist fragwürdig. Die FDA muss sich zwar nicht an die Empfehlungen der Beratungsgremien halten, tut es aber häufig.
Neuer Indikator für Tumorrisiko?

\section{Männer mit langen Zeigefingern haben seltener Prostatakarzinom}

— Britische Forscher haben einen möglichen Indikator für die Entstehung von Prostatakrebs gefunden. In ihre Studie wurden 1.524 Männer mit diagnostiziertem Prostatakarzinom und 3.044 gesunde Kontrollen einbezogen [Rahman AA et al. BJC; doi: 10.1038/sj.bjc.6605986]. Alle Männer sollten anhand von Abbildungen benennen, ob ihre Zeigefinger länger oder kürzer als ihre Ringfinger sind oder gleich lang.Dabei stellte sich heraus, dass Männer, deren Zeigefinger länger als ihre Ringfinger sind, ein um 63\% geringeres Risiko besitzen, ein Prostatakarzinom zu entwickeln als Männer, die kürzere Zeige- als Ringfinger haben. Bei Männern unter 60 Jahren war das Risiko sogar um $87 \%$ reduziert. Die Forscher hoffen, dass ihre Erkenntnisse zusammen mit anderen Faktoren wie familiäre Belastung dazu beitragen, Risiko-Patienten zu identifizieren, um diese regelmäßig zu screenen.

slx

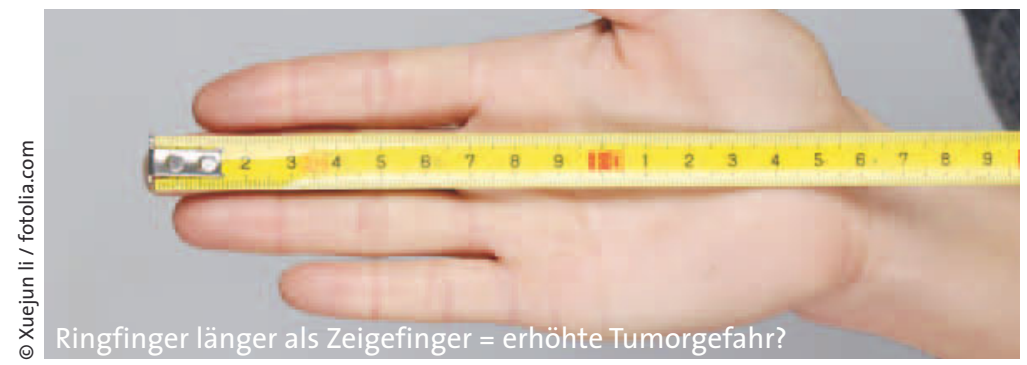

\section{Arztberuf zunehmend verrechlicht DGMR kritisiert KVen}

— Die Deutsche Gesellschaft für Medizinrecht (DGMR) hat die Verrechtlichung des Arztberufs kritisiert. Zunehmend gerieten Ärzte, ,in strafrechtlich relevante Konfliktsituationen“, die sich für sie ,als, unerwartet' darstellen“. Eine „Überdehnung der Straftatbestände“ führe zu „unerwünschten Strafbarkeitsrisiken“, kritisiert der Verband. Dabei gebe es , andere geeignete Sanktionierungsmöglichkeiten im Bereich der ärztlichen Selbstverwaltung“. Daher fordert die DGMR erstens einfachere rechtliche Rahmenbedingungen für Ärzte, etwa in Bezug auf Zulassung, Anstellungsmöglichkeiten und Abrechnung. Für mehr Transparenz könnten auch KVen, Ärztekammern und Krankenkassen sorgen, indem sie zu rechtlich strittigen Fragen öffentlich Stellung nehmen.

Zweitens empfiehlt die DGMR, rechtliche Aspekte verstärkt in die medizinische Ausbildung im Studium einzubeziehen und bei der Zulassung entsprechende Kenntnisse zu verlangen.

Drittens schließlich fordert der Verband eine stärkere Zurückhaltung bei der Strafverfolgung selbst. Es sei davon auszugehen, dass ein Arzt es in der Regel gar nicht wisse, wenn er sich strafbar verhalte. Nur bewusste Verstöße dürften von den KVen an die Staatsanwaltschaft weitergegeben werden, fordert die DGMR. 